\title{
Perspectivas de pesquisa sobre avaliação de desempenho e gerenciamento de projetos
}

\author{
Rogério Tadeu de Oliveira Lacerda \\ Professor do Programa de Pós-graduação em Administração da Universidade Federal de Santa \\ Catarina (UFSC). Bolsista de Produtividade em Desenvolvimento Tecnológico e Extensão Inovadora \\ do CNPq. E-mail: rogerlacerda@gmail.com (Brasil) \\ Lisanda Valim de Oliveira \\ Mestra em Administração pela Universidade Federal de Santa Catarina (UFSC). \\ E-mail: 1valim@terra.com.br (Brasil)

\begin{abstract}
Mayara Lucia Bernardes
Mestranda em Administração na Universidade Federal de Santa Catarina (UFSC). E-mail: mayaralbernardes@gmail.com (Brasil)

Ewerton Sacco Calvetti

Mestrando em Administração na Universidade Federal de Santa Catarina (UFSC). E-mail: escalvetti@gmail.com (Brasil)
\end{abstract}

\section{RESUMO}

Este trabalho tem o objetivo de identificar oportunidades de pesquisa sobre as temáticas de avaliação de desempenho e gerenciamento de projetos. Para isso, foi utilizada a metodologia descrita no processo ProKnow-C (Knowledge Development Process - Constructivist), que permitiu formar um portfólio bibliográfico de 17 artigos relevantes sobre os assuntos escolhidos, demonstrar quantitativamente quais são os de maior destaque, bem como seus autores e periódicos, além de realizar análise sistêmica nesse material para identificar lacunas sobre os temas no meio científico e, assim, instigar a realização de novos trabalhos. O processo de revisão sistêmica foi realizado a partir de lentes (critérios) derivadas da visão de mundo construtivista adotada pelos pesquisadores. Os principais resultados orientam pesquisadores em oportunidades de pesquisa em que a singularidade do contexto e a racionalidade limitada seja levada em conta quando da avaliação de projetos, em contraponto com critérios universais de sucesso em gestão de projetos.

Palavras-chave: Avaliação de desempenho. Análise sistêmica. Construtivismo. Gerenciamento de projetos. Proknow-C. 


\title{
Research perspectives on performance evaluation and project management
}

\begin{abstract}
This work has the objective of identifying research opportunities on the themes of performance evaluation and project management. In order to achieve this goal, the methodology described in ProKnow-C (Knowledge Development Process Constructivist) was used, which allowed the formation of a bibliographic portfolio of 17 relevant articles on the subjects chosen, quantitatively demonstrating which are the most prominent, as well as their authors and periodicals, in addition to performing systemic analysis in this material to identify gaps on the subjects in the scientific environment and, thus, to instigate the accomplishment of new work. The systemic revision process was carried out using criteria derived from the constructivist worldview adopted by the researchers. The main results guide researchers in research opportunities where context uniqueness and limited rationality are taken into account when evaluating projects, as opposed to universal success criteria in project management.
\end{abstract}

Keywords: Performance evaluation. Systemic analysis. Constructivism. Project management. Proknow-C.

\section{Como referenciar em APA:}

Lacerda, R. T. O., Oliveira, L. V., Bernardes, M. L., \& Calvetti, E. S. (2018). Perspectivas de pesquisa sobre avaliação de desempenho e gerenciamento de projetos. R.G.Secr.,GESEC, 9(2). DOI:

\section{Como referenciar em ABNT:}

LACERDA, R. T. O.; OLIVEIRA, L. V.; BERNARDES, M. L.; CALVETTI, E. S. Perspectivas de pesquisa sobre avaliação de desempenho e gerenciamento de projetos. R.G.Secr.,GESEC, v. 9, n. 2, 2018. DOI:
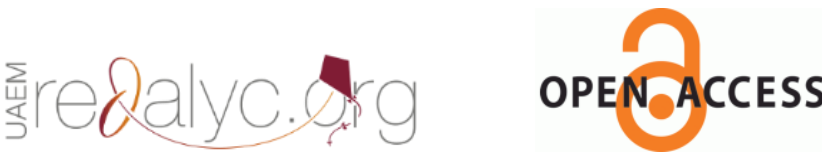


\section{Introdução}

A forma como as organizações medem seu desempenho instiga gestores nas mais diversas áreas. Até o início dos anos de 1980, as mensurações eram baseadas exclusivamente em critérios financeiros. Com o passar do tempo, com mercados mais complexos e dinâmicos, o uso desses critérios não era mais suficiente para avaliar sucesso (Kennerley \& Neely, 2002).

Segundo Behn (2003), a avaliação de desempenho destina-se a atender oito objetivos gerenciais: avaliar, controlar, orçar, motivar, divulgar, celebrar, aprender e melhorar.

Desse modo, a importância do capital intelectual, a fim de, atuar como um diferencial de resposta à mudança com agilidade e flexibilidade vem sendo incorporado a conceitos recentes de avaliação de desempenho (Oliveira, Lacerda, Fiates \& Ensslin, 2016).

A partir deste ponto de vista, para a presente pesquisa, o conceito utilizado para a avaliação de desempenho é de um processo usado para construir conhecimento no decisor, sobre um contex to específico que ele se propõe a avaliar, a partir de sua própria percepção, por meio de atividades que identifiquem, organizem, mensurem ordinalmente e cardinalmente, e integrem os critérios relevantes (Lacerda, L. Ensslin \& S. R. Ensslin, 2012; Lacerda, L. Ensslin, S. R. Ensslin, Knoff \& Martins, 2016; Oliveira et al., 2016).

Consoante com outras áreas de gestão, o gerenciamento de projetos também requer um conjunto relevante de indicadores de desempenho e, igualmente, encontra a mesma dificuldade de escolher modelos de avaliação de acordo com cada contexto (Dweiri \& Kablan, 2006), uma vez que as diferentes situações irão requerer distintos indicadores.

Um dos desafios que os decisores encontram é a avaliação do sucesso dos projetos de sua organização. Até meados da década de 1980, a forma predominante de avaliação era o "triângulo de ferro" (Atkinson, 1999), limitado aos indicadores de tempo, custo e qualidade, conhecidos como critérios hard: critérios objetivos e de mais fácil medição.

A comunidade científica muito tem pesquisado e discutido sobre os critérios e indicadores que definem o sucesso de um produto ou projeto (Cooper, 2007; Jugdev \& Muller, 2006; Lipovetsky, Tishler, Dvir \& Shenhar, 1997; Shenhar \& Dvir, 2007; Tukel \& Rom, 2001). Porém, existem poucos estudos que contribuem de forma reflexiva e crítica a respeito dos processos e metodologias que se focam na construção e uso dos indicadores no contexto de gestão de projetos.

Dentre as publicações que analisam a literatura de gestão de projetos e avaliação de desempenho, pode-se destacar a contribuição de Svejvig e Andersen (2015) que apresenta uma 
reflexão e comparação crítica a respeito da visão clássica de gestão de projetos e o que os autores denominam Rethinking Project Management (RPM). Enquanto na visão clássica de gestão de projeto a eficiência, a padronização, racionalidade perfeita e modelos de mensuração genéricos são ostensivamente utilizados, na RPM os projetos constituem-se em organizações temporárias dentro de contextos complexos, incertos e conflituosos de interesses. Na RPM, a racionalidade limitada é considerada como um fato sugerindo a incorporação da percepção dos stakeholders para uma construção de conhecimento e buscar modelos adaptativos e singulares ao contexto no qual se estabelece o projeto em questão. Assim, onde os modelos tradicionais buscam critérios e indicadores universais, na RPM busca-se a singularidade de indicadores (Packendorff, 1995; Jugdev, Thomas \& Delisle, 2001; Winter, Smith, Morris \& Cicmil, 2006; Shenhar \& Dvir, 2007; Andersen, 2008; Lenfle \& Loch, 2010).

Na revisão bibliográfica de Svejvig e Andersen (2015), os resultados apontam que a RPM chama a atenção da comunidade científica e indica um aumento considerável de publicações desde 2006, explicitando claramente as limitações das visões clássicas de gestão de projetos que se foca modelos genéricos de avaliação ("boas práticas") e ênfase na racionalidade perfeita dos gestores (objetividade). Na abordagem clássica de gestão de projetos, minimiza-se ou até aponta como uma fragilidade a aprendizagem fruto da ação e reflexão no contexto singular do projeto (subjetividade). Como exemplo dessa dominância, a revisão bibliográfica de Willems e Vanhoucke (2015) destaca que 85\% de todos os artigos analisados que se focam em controle de projetos usam elementos quantitativos e podem ser rotulados de determinísticos, estocásticos ou fuzzy; e 90\% dos artigos dessa mesma amostra fornecem procedimentos de testes baseados em simulações e/ou dados históricos.

Por outro lado, na RPM, a subjetividade (valores e preferências dos gestores) é um ponto fundamental a ser considerado nos processos de avaliação de desempenho, sendo essa uma evolução da visão clássica, mas não descartando a parte objetiva dos sistemas de mensuração (Pollack, 2007; Williams, 2005; Lacerda, Ensslin L. \& Ensslin S., 2011). Sendo assim, a presente pesquisa beneficia-se da oportunidade de pesquisa das proposições de Svejvig e Andersen (2015) a respeito de pesquisas críticas em RPM.

Outra revisão bibliográfica relevante a ser destacada a respeito dos temas deste artigo é a contribuição de Ahmad, Svalestuen, Andersen, \& Torp (2016) que explicitam como resultados a ênfase de publicações a respeito de indicadores laggers que avaliam os resultados e impacto do projeto após a execução de atividades. Porém, essa revisão destaca a relevante oportunidade de pesquisa em métodos que criam modelos de avaliação de projetos valendo-se 
de indicadores drivers, que apoiam a decisão durante as faces de execução do projeto, reconhecendo a importância da retroalimentação de ação e reflexão da equipe do projeto e indo além da visão econômica dos indicadores de avaliação de desempenho de projeto (Ahmad et. al., 2016; Willems \& Vanhoucke, 2015; Ngacho \& Das, 2015). Essas oportunidades de pesquisa são aproveitadas neste artigo.

Diante desse cenário, emerge a motivação que levou os autores deste trabalho a querer proceder com a seguinte investigação: a partir da análise de conteúdo de artigos científicos relevantes sobre avaliação de desempenho e gerenciamento de projetos, quais são as oportunidades de pesquisa identificadas nestes temas?

Para o alcance do propósito mencionado, foi utilizado o ProKnow-C como método para gerar conhecimento no pesquisador sobre os temas (Lacerda, L. Ensslin \& S. R. Ensslin, 2014; Lacerda et al., 2016). Tal abordagem iniciou com um processo estruturado para a seleção do conjunto relevante de artigos, realização de bibliometria no conteúdo selecionado e culminou com a análise sistêmica desse material.

Em nível de abordagem escolhida pelos autores, as atividades mencionadas desenvolveram-se a partir de uma visão construtivista, ou seja, amparadas na premissa de que o decisor precisa expandir sua compreensão para os impactos de suas decisões dentro das perspectivas que ele julgar serem relevantes e, embasado neste conhecimento desenvolvido, ele deve avaliar diferentes aspectos e propor as melhorias (Marafon, L. Ensslin, Lacerda \& S. R. Ensslin, 2015).

Na visão adotada, a operacionalização da avaliação de desempenho foi norteada por seis lentes que guiaram a análise de conteúdo dos artigos do portfólio: filiação teórica, singularidade, identificação dos critérios, formas de mensuração, integração das escalas e gestão. Elas são detalhadas nos capítulos que seguem.

Após esta introdução, o restante deste artigo está estruturado em: explanação sobre a metodologia utilizada nas três fases que compuseram este trabalho: seleção dos artigos, análise bibliométrica e análise sistêmica; depois são apresentados os resultados da análise do portfólio bibliográfico à luz dos pressupostos que orientaram esta pesquisa; em seguida são expostas as conclusões e oportunidades para futuros estudos; e, por fim, as referências deste documento são listadas. 


\section{Metodologia}

A seguir está descrito o enquadramento metodológico da pesquisa.

\section{Enquadramento metodológico}

Esta pesquisa assume um caráter descritivo pois, além do processo, descreve certos atributos dos artigos selecionados (Gil, 1999). O procedimento técnico é a pesquisa bibliográfica, por utilizar-se de documentos científicos. Os dados utilizados são obtidos a partir de fontes secundárias (publicações científicas), materiais esses que já foram previamente trabalhados por outros profissionais da academia.

A essência do trabalho é teórica, mas, os resultados desta pesquisa são de cunho aplicado, pois a seleção final dos artigos mais relevantes será subsídio para o desenvolvimento de futuros trabalhos pelos pesquisadores. Ou seja, contribuirá para fins práticos, buscando soluções para problemas concretos (Cervo, Bervian \& Silva, 2007).

Em relação ao instrumento utilizado, optou-se pelo uso do ProKnow-C (Knowledge Development Process - Constructivist), método criado por pesquisadores do LabMCDA. (Marafon, L. Ensslin, S. R. Ensslin \& Lacerda, 2012; Lacerda et al., 2012; Lacerda, L. Ensslin \& S. R. Ensslin, 2011a; Silva da Rosa, S. R. Ensslin, L. Ensslin \& João Lunkes, 2012; S. R. Ensslin, L. Ensslin, Lacerda, \& Dos Santos Matos, 2013; Knoff, Lacerda, L. Ensslin, \& S. R. Ensslin , 2014).

\section{Processo de seleção dos artigos}

Em abril de 2015, interessados em conhecer a literatura científica sobre os temas gerenciamento de projetos e avaliação de desempenho, os pesquisadores autores deste trabalho iniciaram um processo estruturado para seleção de artigos relevantes.

Esse procedimento iniciou pela escolha das bases de dados que seriam utilizadas; no caso foram a Web of Science (antiga ISI) e Scopus. A primeira por dar origem ao JCR (Journal Citation Report) que é parâmetro para indicação do fator de impacto de periódicos (Knoff et $a l ., 2014)$ e a segunda, por ser a maior base de dados de resumos e citações: revistas científicas, livros e anais de congressos (Elsevier, 2015).

Em seguida, foi necessário definir as palavras-chaves que seriam usadas nas buscas. Para o eixo de pesquisa "avaliação de desempenho", foram utilizadas as mesmas palavras-chaves 
utilizadas por estudos bibliométricos anteriores sobre este assunto (Lacerda et al., 2012; Ensslin et al., 2013; Dienstmann, Lacerda, L. Ensslin \& S. R. Ensslin, 2014), que são: assessment, evaluation, measure e appraisal. Já para o eixo "gerenciamento de projetos", foram necessários alguns testes e leituras não estruturadas, até se alcançar o consenso quanto ao uso das palavras "Project success" e "Project performance", além de "Project management". No Quadro 1, é possível verificar a lista final de combinações utilizadas nesta pesquisa.

Quadro 1 - Palavras-chave da pesquisa.
\begin{tabular}{|l|l|l|}
\hline \multicolumn{3}{|c|}{ Combinações de Palavras-chave } \\
\hline EIXO 1 & & EIXO 2 \\
\hline Appraisal & AND & "Project Management" \\
\hline Assessment & AND & "Project Management" \\
\hline Evaluation & AND & "Project Management" \\
\hline Measure & AND & "Project Management" \\
\hline Appraisal & AND & "Project Performance" \\
\hline Assessment & AND & "Project Performance" \\
\hline Evaluation & AND & "Project Performance" \\
\hline Measure & AND & "Project Performance" \\
\hline Appraisal & AND & "Project Success" \\
\hline Assessment & AND & "Project Success" \\
\hline Evaluation & AND & "Project Success" \\
\hline Measure & AND & "Project Success" \\
\hline
\end{tabular}

Fonte: (Oliveira et al., 2016, p.101)

O horizonte temporal dos artigos foi de dez anos desde sua publicação, seguindo parâmetros de artigos destacados da área como Wernerfelt (1995), Barney, Wright, Ketchen \& David (2001), Lowe \& Gardner (2001), Taticchi, Tonelli \& Cagnazzo (2010), Klijn (2008) e Lacerda et al. (2012). Os autores dessa pesquisa entendem que esse horizonte temporal é adequado, uma vez que indicadores de publishers para meia-vida de uma publicação ou periódico tem uma saturação em dez anos.

A partir da definição das palavras-chave, as buscas retornaram 6.920 artigos que passaram por etapas de: eliminação de redundância (documentos repetidos); alinhamento baseado na leitura do título; priorização de artigos com maior reconhecimento científico - para tal foi utilizado o número de citações exibido pela ferramenta online Google Scholar (2015) que possui, entre seus propósitos, a finalidade de agrupar documentos acadêmicos, levando em conta o texto integral, onde foram publicados, quais são os autores e ainda o número e tipo de citações em outras publicações acadêmicas -; e, alinhamento baseado na leitura do resumo do artigo. Nesta etapa, havia 40 documentos que tiveram uma leitura integral e, então, foi possível chegar à lista final dos 17 artigos que compõem o portfólio bibliográfico.

As referências bibliográficas dos artigos dos últimos dez anos que foram selecionados 
para subsidiar o referencial teórico do assunto avaliação de desempenho e gerenciamento de projetos são:

1. Andersen, E. S., et. al. (2006). "Exploring project success." Baltic Journal of Management 1(2): 127-147.

2. Barclay, C. (2008). "Towards an integrated measurement of IS project performance: The project performance scorecard." Information Systems Frontiers 10(3): 331-345.

3. Barclay, C. and K.-M. Osei-Bryson (2010). "Project performance development framework: An approach for developing performance criteria \& measures for information systems (IS) projects." International Journal of Production Economics 124(1): 272-292.

4. Blindenbach-Driessen, F., et. al. (2010). "Subjective performance assessment of innovation projects." Journal of Product Innovation Management 27(4): 572-592.

5. Cao, Q. and J. J. Hoffman (2011). "A case study approach for developing a project performance evaluation system." International Journal of Project Management 29(2): 155-164.

6. Jha, K. N. and K. C. Iyer (2007). "Commitment, coordination, competence and the iron triangle." International Journal of Project Management 25(5): 527-540.

7. Karlsen, J. T., et. al. (2005). "What characterizes successful it projects." International Journal of Information Technology \& Decision Making 4(4): 525-540.

8. Ling, F. Y. Y. et. al. (2009). "Key project management practices affecting Singaporean firms' project performance in China." International Journal of Project Management 27(1): 59-71.

9. Marques, G. et. al. (2011). "Multi-criteria performance analysis for decision making in project management." International Journal of Project Management 29(8): 1.057-1.069.

10. Mir, F. A. and A. H. Pinnington (2014). "Exploring the value of project management: Linking project management performance and project success." International Journal of Project Management 32(2): 202-217.

11. Mota, C. M. D. M. et. al. (2009). "A multiple criteria decision model for assigning priorities to activities in project management." International Journal of Project Management 27(2): 175-181.

12. Nassar, N. and S. Abourizk (2014). "Practical application for integrated 
performance measurement of construction projects." Journal of Management in Engineering 30(6).

13. Oliveira Lacerda, R. T. de, et. al. (2011). "A performance measurement view of IT project management." International Journal of Productivity and Performance Management 60(2): 132-151.

14. Qureshi, T. M. et. al. (2009). "Significance of project management performance assessment (PMPA) model." International Journal of Project Management 27(4): 378388.

15. Rosacker, K. M. and D. L. Olson (2008). "An empirical assessment of it project selection and evaluation methods in state government." Project Management Journal 39(1): 49-58.

16. Thomas, G. and W. Fernández (2008). "Success in IT projects: A matter of definition?" International Journal of Project Management 26(7): 733-742.

17. Xu, Y. and C. H. Yeh (2014). "A performance-based approach to project assignment and performance evaluation." International Journal of Project Management 32(2): 218-228.

\section{Resumo da análise bibliométrica}

Seguindo as etapas do processo ProKnow-C (L. Ensslin, S. R. Ensslin, Lacerda \& Tasca, 2010), fez parte deste estudo a realização de uma bibliometria. Esse tipo de técnica, muito mais do que um levantamento estatístico puro e simples, permite a realização de análises complexas e diversificadas sobre as atividades de produção e comunicação científica (Ferreira, 2012).

Tal atividade pode ser separada em três fases distintas e complementares: análise dos 17 artigos que compõem o portfólio bibliográfico; análise das referências mencionadas nestes artigos e, por fim, a análise combinada desse conjunto de informações (portfólio e suas referências). Em todas elas, as principais variáveis analisadas foram a representatividade dos periódicos, dos autores e a relevância deles, mensurados a partir do número de citações do Google Scholar.

No que se refere aos periódicos, foi possível identificar que o de maior destaque é o International Journal of Project Management, responsável por mais de 50\% das publicações nos tópicos combinados desta pesquisa em todas as análises realizadas.

Com referência aos autores do portfólio bibliográfico, salienta-se Corlane Barclay da 
Universidade de Tecnologia da Jamaica, que é responsável por dois dos 17 artigos. Os demais autores contribuíram com um artigo cada.

Para identificação dos artigos mais relevantes do portfólio, foi realizado um cruzamento de dados que considera o número de citações dos artigos do portfólio e o número de citações de outros artigos - entre todos mencionados nas referências - escritos por autores do portfólio bibliográfico. Tal levantamento leva aos seis artigos de destaque assim caracterizados.

O artigo de Thomas e Fernandez (2008), possui considerável destaque na comunidade acadêmica. Já os dois artigos, Jha e Iyer (2007) e Ling, Low, Wang e Lim (2009), além de destaque, foram criados por autores que possuem outras relevantes publicações nas referências deste estudo.

Por fim, os três artigos, Lacerda, L. Ensslin e S. R. Ensslin (2011b), Mota, Almeida e Alencar (2009) e Nassar e Abourizk (2014) ganharam destaque pelo fato de algum de seus autores - nomeadamente Ensslin, L.; Almeida, A. T. e AbouRizk, S. M. possuírem publicações relevantes mencionadas nas referências.

\section{Processo de análise sistêmica}

Tomando por base o processo de análise sistêmica definido no ProKnow-C, realiza-se a análise crítica dos 17 artigos do portfólio a partir de pressupostos - chamados "lentes" - da afiliação teórica de avaliação de desempenho, com o objetivo de identificar lacunas para futuras investigações (Ensslin et al., 2010). No meio acadêmico, esse processo de revisão aumenta o rigor do trabalho, além do seu propósito principal que é o de prover insights coletivos por meio da síntese teórica em determinados campos (Tranfield, Denyer \& Smart, 2003).

No presente artigo foi utilizada a abordagem construtivista como visão de mundo escolhida pelos pesquisadores. Isso significa afirmar que o decisor possui papel fundamental, pois ele é a pessoa que tem a responsabilidade e a autoridade necessárias para a tomada de decisão na organização (Ensslin et al., 2010; Lacerda, Klein, Fulco, Santos \& Bittarello, 2017). É a partir do sistema de valores e preferências dele, que o modelo de avaliação será construído (Ensslin et al., 2010; Lacerda et.al., 2017). O facilitador do processo de avaliação de desempenho tem a função de construir conhecimento no decisor e pode fazer uso das ferramentas que julgar mais convenientes para que o decisor compreenda os impactos da situação vigente em seu próprio sistema de valores, bem como a evolução causada por suas decisões nos objetivos da organização (Roy, 1993). 
Cabe ressaltar ainda que, em um modelo construtivista, o decisor - sempre que possível - tenta favorecer os intervenientes do processo, pois uma vez que eles participam do contexto, é importante contar com sua colaboração (Keeney, 1992). Portanto, o decisor precisará compreender as consequências de suas decisões e, em alguns casos, justificar ou alterar seu posicionamento, em função da participação desses intervenientes no desenvolvimento do modelo (Lacerda et. al. , 2012).

Outra característica relevante desta abordagem é que, no construtivismo, as incoerências entre discurso e prática são vistas de forma positiva, pois essas divergências servirão para gerar conhecimento (Ensslin et al., 2010). Além disso, a abordagem construtivista reconhece a singularidade do contexto, ou seja, os trabalhos realizados sob essa perspectiva, só são aplicados no contexto que foram criados, uma vez que os modelos são construídos com base nos valores e preferências de um decisor específico (Lacerda et al., 2012).

Vale ressaltar que o construtivismo também possui suas limitações. Pode-se citar o fato de que ele exige muitas interações entre facilitador, decisor e intervenientes; é imprescindível o engajamento e legitimação por parte do real decisor na organização; e ainda, não é possível uma replicação do modelo, uma vez que alterado o decisor ou o contexto, o modelo necessariamente requer revisão (Roy, 1993).

Tomando por base o paradigma construtivista mencionado e a perspectiva da avaliação de desempenho, foram utilizadas lentes de análise que permitem analisar os procedimentos realizados pelos pesquisadores nos trabalhos do portfólio bibliográfico aqui estudado. $\mathrm{O}$ termo "lentes" faz referência às lentes de Brunswik mencionadas no trabalho de Hammond e Stewart (2001) e explicitadas no Quadro 2. análise.

Quadro 2 - Lentes usadas para representar o conceito de avaliação de desempenho adotado na

\begin{tabular}{|l|l|l|}
\hline & LENTES & O que busca? \\
\hline 1 & Conceito & Qual a filiação teórica? \\
\hline 2 & Singularidade & Reconhece que o problema é único? \\
\hline 3 & Processo para Identificar & $\begin{array}{l}\text { Tem processo para identificar os objetivos } \\
\text { segundo a percepção do decisor? }\end{array}$ \\
\hline 4 & Mensuração & $\begin{array}{l}\text { Reconhece que os descritores (indicadores de } \\
\text { desempenho) são escalas ordinárias? }\end{array}$ \\
\hline 5 & Integração & Reconhece que a integração requer níveis de referência? \\
\hline & Gestão & O conhecimento gerado permite conhecer o perfil atual, \\
\end{tabular}

Fonte: (Lacerda et al., 2014, p.165)

R.G. Secr., GESEC, São Paulo, v. 9, n. 2, p 96-125, mai./ago. 2018. 


\section{Enquadramento dos artigos analisados}

Com a relação dos artigos a serem analisados e já definidas as lentes que embasam essa atividade, o próximo passo é o enquadramento dos artigos para cada uma dessas perspectivas.

\section{Considerações lente 1: abordagem (filiação teórica)}

A relevância da abordagem utilizada no estudo diz respeito aos filtros utilizados pelos pesquisadores, fazendo-os perceber certas coisas do contexto em que atuam e ignorando outras (Melão \& Pidd, 2000).

Ao todo, foram identificados sete artigos que utilizam uma abordagem descritivista: Karlsen, Andersen, Birkely e Odegard (2005), Andersen, Birchall, Jessen e Money (2006), Jha e Iyer (2007), Ling et al. (2009), Rosacker e Olson (2008), Thomas e Fernández (2008) e Xu e Yeh (2014). Esses modelos tomam por base os desempenhos passados (histórico) para embasar suas previsões futuras. Na pesquisa de Rosacker e Olson (2008) eles enviaram questionários para órgão públicos responderem quanto aos métodos que usaram em seus projetos, bem como os resultados obtidos e, a partir das respostas e aplicação de técnicas estatísticas, identificaram relacionamentos entre as variáveis que entendem ser aplicáveis em todos os contextos.

A segunda presença mais significativa foi de seis artigos que utilizam abordagem prescritivista: Barclay e Osei-Bryson (2010), Barclay (2008), Marques, Gourc e Lauras (2011), Nassar e Abourizk (2014), Mota et al. (2009) e Cao e Hoffman (2011). Nessa abordagem, a solução mais apropriada é prescrita pelo pesquisador, mas são os valores dos decisores que mais importam e, por isso, esse é um exemplo de apoio à decisão. No trabalho de Mota et al. (2009), eles utilizam a metodologia MCDA (Multiple Criteria Decision Analysis) para apoiar o decisor a identificar as tarefas mais relevantes do seu modelo de gerenciamento de projetos.

Foram identificados três trabalhos que utilizaram abordagens normativistas: Blindenbach-Driessen, Van Dalen e Van Den Ende (2010), Mir e Pinnington, (2014) e Qureshi, Warraich e Hijazi (2009), sendo que os últimos dois se valeram do modelo PMPA (Project Management Performance Assessment) construído por Bryde (2003) para desenvolver suas pesquisas. Ou seja, são trabalhos baseados na racionalidade, em que se aceita que a realidade está adequadamente representada pelo modelo teórico definido pelo pesquisador e os desvios aos resultados são considerados decisões equivocadas. Salienta-se que, nesta abordagem, não é dado espaço ao decisor para colocar sua opinião. 
As abordagens descritivista e normativista são utilizadas para a tomada de decisão, ou seja, elas consideram apenas o objeto para o contexto decisório. São também chamados de modelos "realistas", segundo Roy (1993). Nesses casos, a tomada de decisão é guiada pelas generalizações assumidas nesses constructos e um dos pontos desfavoráveis é que, pelo fato dos critérios não terem vindo dos decisores, isso permite que eles se isentem da responsabilidade dos resultados do modelo.

Apenas um artigo foi identificado como utilizando uma abordagem construtivista: Lacerda et al. (2011b). A principal característica nesse modelo de apoio à decisão é que o pesquisador deseja construir conhecimento no decisor. E o decisor, por sua vez, sempre que possível, deseja favorecer também as partes envolvidas no processo.

Uma característica do trabalho construtivista em relação aos demais, é que as incoerências existentes entre discurso e prática, identificadas durante a construção do modelo, são vistas de forma positiva, pois contribuem no processo de geração de conhecimento (Roy, 1993). Por outro lado, o fato de ele depender da legitimação do decisor também pode ser considerado como uma limitação para sua aplicação.

A partir dessas análises, podemos perceber que a literatura vigente ainda carece de mais pesquisas utilizando abordagens construtivistas, uma vez que elas trazem o ganho de gerar conhecimento no decisor, aumentando seu engajamento com a solução por ele identificada (com o apoio do facilitador). Isso é particularmente relevante em projetos de inovação, onde há limitação de conhecimento no gestor, gerando incertezas de várias ordens, desde escopo, estimativas dentre outros.

\section{Considerações lente 2: reconhecimento da singularidade}

Os aspectos avaliados em um dado projeto representam os objetivos que operacionalizam os valores percebidos pelos decisores como necessários e suficientes para o seu gerenciamento naquele momento específico, a partir de uma visão construtivista (Roy, 1993). A partir deste conceito, pode-se depreender que são as escolhas dos decisores, que determinam a arquitetura de negócios da organização, que por sua vez é o que a tornará difícil de imitar e, portanto, diferenciada das demais (Teece, 2007).

A análise dos artigos do portfólio baseia-se na identificação de que foram incorporados aspectos do contexto da organização em que o gerenciamento é realizado e foi identificado um equilíbrio na quantidade de trabalhos que reconhecem o paradigma da singularidade e os que a

R.G. Secr., GESEC, São Paulo, v. 9, n. 2, p 96-125, mai./ago. 2018. 
ignoram.

Os artigos Karlsen et al. (2005), Andersen et al. (2006), Jha e Iyer, (2007), BlindenbachDriessen et al. (2010), Ling et al. (2009), Mir e Pinnington (2014), Qureshi et al. (2009), Rosacker e Olson (2008) e Thomas e Fernández (2008) trabalham com modelos universais, ou seja, assumem que as organizações são iguais e não há peculiaridades entre elas.

Enquanto que os artigos Barclay e Osei-Bryson (2010), Barclay (2008), Marques et al. (2011), Nassar e Abourizk (2014), Mota et al. (2009), Lacerda et al. (2011b), Xu e Yeh (2014) e Cao e Hoffman (2011) entendem que o gestor e os recursos fazem parte do contexto onde as decisões são tomadas, ou seja, eles reconhecem o paradigma da singularidade.

Os modelos genéricos podem também ser chamados de objetivistas, por ignorarem o gestor e as singularidades de cada organização. Entende-se que esta é uma limitação desta visão, pois assume as premissas de que o gestor não faz parte do contexto em que as decisões são tomadas e que as organizações são iguais (L. Ensslin, Back, S. R. Ensslin e Lacerda, 2013).

Essa afirmação carrega consigo um importante elemento para projetos que são financiados com a expectativa de trazer diferenciais competitivos para a organização, que precisam da singularidade do contexto para se distanciar de soluções genéricas, copiadas ostensivamente por concorrentes e outros atores do mercado (Lacerda et al., 2014).

\section{Considerações lente 3: como identifica os critérios}

O objetivo desta lente é entender como os pesquisadores construíram os critérios utilizados em seus modelos de avaliação de desempenho. Apenas um dos trabalhos (Xu \& Yeh, 2014), não explicitou como essa atividade foi elaborada.

Uma maioria representativa dos artigos (11 de 17 artigos) utilizou-se da revisão da literatura vigente para definir as variáveis de desempenho analisadas em seus constructos. São eles: Karlsen et al. (2005), Andersen et al. (2006), Jha e Iyer (2007), Blindenbach-Driessen et al. (2010), Barclay (2008), Marques et al. (2011), Ling et al. (2009), Mir e Pinnington (2014), Qureshi et al. (2009), Rosacker e Olson (2008) e Thomas e Fernández (2008).

Há, também, três artigos que definiram seus critérios de análise com base nos valores e preferências dos decisores: Mota et al. (2009), Lacerda et al. (2011b) e Cao e Hoffman (2011).

Quanto aos dois artigos remanescentes, um deles Barclay e Osei-Bryson (2010) tomou por base a percepção das partes interessadas no projeto; enquanto o outro Nassar e Abourizk (2014) preferiu consultar especialistas da área de gestão de projetos para definir os critérios de 
avaliação de desempenho mais apropriados.

A análise deste tópico evidencia o uso da literatura científica vigente como principal entrada dos modelos de avaliação de desempenho. Tal conclusão gera a reflexão quanto à validade e legitimação desses modelos, uma vez que suas premissas fundamentais não estão amparadas na perspectiva daquele que define as escolhas da organização, no caso o tomador de decisão.

Essa característica de uso primordial da literatura demonstra especial fragilidade quando empregada em trabalhos que reconhecem a singularidade de seus modelos. Ou seja, os pesquisadores aceitam que seus modelos são únicos, porém o basearam em critérios genéricos.

\section{Considerações lente 4: como as escalas são construídas (mensuração)}

Outro dos critérios avaliados neste estudo diz respeito à forma como que o desempenho é medido nos artigos estudados. Dentre os dezessete trabalhos, cinco deles não explicitaram como suas escalas foram construídas: Andersen et al. (2006), Barclay e Osei-Bryson (2010), Barclay (2008), Mir e Pinnington (2014) e Xu e Yeh (2014).

A escala cujo uso é mais frequente, e de uma forma ou outra é utilizada em todos artigos, é a escala ordinal, aquela que estabelece uma preferência de ordem. Porém, cabe salientar algumas particularidades em sua utilização, tal como o fato de que cinco dos trabalhos Karlsen et al. (2005), Blindenbach-Driessen et al. (2010), Ling et al. (2009), Qureshi et al. (2009) e Rosacker e Olson (2008) valeram-se do uso da escala de Likert, que é uma forma de mensuração comumente usada em questionários para as pessoas se manifestarem sobre sua propensão a um dado objetivo e/ou questão. Apesar de sua facilidade de construção, esta escala não atende a todas as propriedades necessárias para ser considerada respaldada cientificamente: mensurabilidade; operacionalidade; homogeneidade; inteligibilidade; permitir distinção entre desempenho melhor e pior; e, respeitar as propriedades das escalas ordinais (Barzilai, 2001).

Em quatro trabalhos, foi utilizada simplesmente a escala ordinal. São eles: Jha e Iyer, (2007), Thomas e Fernández (2008), Mota et al. (2009) e Cao e Hoffman (2011).

Os artigos Marques et al. (2011), Nassar e Abourizk (2014) e Lacerda et al. (2011b) valeram-se do aprimoramento da conversão da escala ordinal em cardinal. Tal iniciativa traz a vantagem de que, somente assim, torna-se viável uma adequada comparação entre distintos indicadores.

A necessidade de respeitar os limites das escalas ordinais utilizando-as somente para 
operações matemáticas de frequência, contagem, mediana e moda, indica que há oportunidade para incrementar trabalhos que explorem mais esse processo de transformação de escalas ordinais em cardinais. Essa é a alternativa viável para permitir a comparação dos diferentes níveis de desempenho e isso é importante para que se possa ampliar o entendimento do contexto em que a organização está inserida.

\section{Considerações lente 5: como o modelo é integrado}

A integração dos indicadores é premissa para uma análise sistêmica da situação atual da organização e seu contexto, bem como para permitir ações de aperfeiçoamento neste meio.

A maior representatividade dos trabalhos - seis deles - executou a integração utilizando modelos estatísticos, mais precisamente o uso de correlações. São os artigos: Andersen et al. (2006), Blindenbach-Driessen et al. (2010), Ling et al. (2009), Mir e Pinnington (2014), Qureshi et al. (2009) e Rosacker e Olson (2008).

Há cinco trabalhos neste portfólio que não explicitaram a questão da integração das escalas em suas pesquisas: Karlsen et al. (2005), Jha e Iyer (2007), Barclay e Osei-Bryson (2010), Barclay (2008) e Thomas e Fernández (2008).

Dois dos trabalhos analisados mencionaram que utilizaram taxas de compensação para fazer a integração dos indicadores estudados: Mota et al. (2009) e Lacerda et al. (2011b).

Outros dois artigos utilizaram DEA: Xu e Yeh (2014) e Cao e Hoffman (2011). DEA significa Data Envelopment Analysis e é um método não paramétrico para avaliar a eficiência relativa de um conjunto de unidades de tomada de decisão (Charnes, Cooper, \& Rhodes, 1978). Resumidamente, essa técnica faz a integração das escalas a partir de dados históricos com o objetivo de maximizar os resultados futuros.

O trabalho Marques et al. (2011) valeu-se exclusivamente da matemática para integrar suas escalas, mais precisamente do uso de pesos.

O método AHP (Analytic Hierarchy Process) é um método de avaliação baseada na comparação de pares a partir de critérios definidos por especialistas para estabelecer suas escalas principais (Saaty, 2008) e foi utilizado apenas no artigo Nassar e Abourizk (2014). A base desse método é a análise das alternativas disponíveis, diferente de outros que trabalham sobre objetivos. O método possui algumas limitações, dentre as quais cabe destacar que ele não proporciona oportunidade de melhorias, apenas faz a avaliação das opções baseado nos critérios predefinidos. 
Observa-se que dentre os modelos de integração utilizados, apenas três deles chegaram a um índice global de desempenho: Nassar e Abourizk (2014), Mota et al. (2009) e Lacerda et al. (2011b); os demais permitem apenas a análise critério a critério. Tal fato permite inferir que há a possibilidade de mais estudos que aceitem a integração de indicadores, pois somente assim é possível identificar o nível global de desempenho para informar ao decisor onde a organização se encontra dentro do contexto avaliado, bem como para saber qual é o critério que mais contribui para tal indicador. Esta análise faz emergir as possibilidades de melhoria que serão apreciadas na próxima lente.

Além disso, também há a oportunidade de novos estudos que explorem a mensuração de objetivos definidos pelo decisor ao invés das alternativas disponíveis em dada circunstância; e ainda, o reconhecimento quanto à necessidade de níveis de referência para a comparação par a par dos indicadores.

\section{Considerações lente 6: como diagnostica e propõe melhorias (gestão)}

Esta análise visa identificar o status quo, ou seja, qual a situação de cada um dos indicadores em dado momento. Entretanto, o presente portfólio trouxe a característica de que aproximadamente a metade dos trabalhos utilizaram dados post mortem, ou seja, valeram-se de informações de projetos já encerrados. Isso aconteceu porque os autores usaram as pesquisas em campo para definir, priorizar e identificar relações dos critérios com a performance dos projetos. Por exemplo, os artigos Ling et al. (2009) e Thomas e Fernández (2008) utilizaram o diagnóstico para identificar boas práticas já utilizadas na gestão de projetos, ou seja, os critérios que mais interferiram na percepção de sucesso dos projetos executados. Já o artigo Blindenbach-Driessen et al. (2010), identificou quais modelos de avaliação sofreram menos viés em suas respostas.

A variação desta análise post mortem diz respeito ao objetivo do diagnóstico e com quem ele foi feito. Dentre os oito trabalhos que usaram esse tipo de informação, alguns deles utilizaram questionários com os stakeholders: Jha e Iye (2007), Barclay (2008), Ling et al. (2009), Mir e Pinnington (2014) e Qureshi et al. (2009); enquanto outros basearam o diagnóstico em questionários com os gerentes do projeto: Blindenbach-Driessen et al. (2010), Rosacker e Olson (2008) e Thomas e Fernández (2008).

Os dois artigos Karlsen et al. (2005) e Barclay e Osei-Bryson (2010) não abordam a questão do diagnóstico em suas pesquisas. 
Perspectivas de pesquisa sobre avaliação de desempenho e gerenciamento de projetos

Em cinco trabalhos, os autores diagnosticaram a situação atual de uma maneira quantitativa, são eles: 1) Andersen et al. (2006) e Marques et al. (2011) utilizaram modelos matemáticos ou estatísticos; 2) Nassar e Abourizk (2014) valeram-se do método AHP; e, 3)Xu e Yeh (2014) e Cao e Hoffman (2011) fizeram uso da técnica DEA.

Já os artigos Mota et al. (2009) e Lacerda et al. (2011b), realizaram o diagnóstico em suas pesquisas por meio de modelos qualitativos e quantitativos em que metas foram definidas e comparadas com a situação atual.

A limitação mais evidente neste levantamento é a quantidade de trabalhos que utilizaram apenas informações de projetos já encerrados, caracterizando assim que eles não suportam o apoio à decisão, uma vez que não permitirão a melhoria da situação atual. Esses estudos, na visão de Roy (1993), podem ser considerados como tomada de decisão, pois funcionam como input para abordagens normativistas - na medida que gerarem modelos que sejam legitimados cientificamente - ou para abordagens descritivistas, que se valem de resultados histórico para prever o futuro.

Pode-se comentar também que há a oportunidade de mais estudos que utilizem critérios qualitativos - além dos quantitativos - para propiciar reflexão e construção de conhecimento no decisor e, assim, ele possa visualizar as consequências estratégicas de suas ações.

Por fim, ainda na lente de gestão, foram observados como os trabalhos deste portfólio contribuem para aperfeiçoar o contexto estudado. A primeira constatação é que dentre os 17 artigos estudados, 10 deles não abordaram as oportunidades de melhoria.

Em quatro dos artigos: Mota et al. (2009), Lacerda et al. (2011b), Xu e Yeh (2014) e Cao e Hoffman (2011); os autores propõem a aplicação de ferramentas que servirão para definir, ordenar e revisar os critérios a serem mensurados, bem como a priorização de ações mais apropriadas dados os objetivos.

Há, ainda, três trabalhos Marques et al. (2011), Nassar e Abourizk (2014) e Thomas e Fernández (2008) que indicam o que podemos chamar de ciclo PDCA, uma vez que contemplam a definição de indicadores, a coleta dos dados e sua frequente monitoria; gerando um processo de aprendizado advindo da execução dos planos e dando um entendimento maior da situação pelos decisores por meio da ação estratégica.

A etapa de monitoramento utilizada no ciclo PDCA, ainda que imprescindível, pode ser limitada caso não sejam utilizadas ferramentas que permitam a ampliação do entendimento das consequências das ações nos objetivos estratégicos dos decisores antes da sua execução, aumentando assim o risco de investimentos em questões de baixa relevância para a organização. 
Salienta-se também que o expressivo número de artigos que não propõem melhorias conduz à conclusão de que esta é uma das oportunidades de estudo propiciada por esta análise.

\section{Síntese e oportunidades identificadas a partir da análise sistêmica}

A partir dos levantamentos realizados na etapa de análise sistêmica de revisão do portfólio bibliográfico, as oportunidades de pesquisa foram identificadas e listadas no Quadro 3:

\begin{tabular}{|c|c|}
\hline Lente & Análise a ser realizada \\
\hline Abordagem & $\begin{array}{l}\text { Como elaborar um modelo de avaliação de } \\
\text { desempenho que considere os valores e preferências } \\
\text { dos decisores ao mesmo que tempo que gera } \\
\text { conhecimento neles? }\end{array}$ \\
\hline Singularidade & $\begin{array}{l}\text { Como elaborar um modelo de avaliação de } \\
\text { desempenho que permita a combinação das } \\
\text { considerações subjetivas dos decisores com os } \\
\text { aspectos físicos e humanos do ambiente que está } \\
\text { inserido em um modelo único para apoiar a gestão } \\
\text { estratégica da organização? }\end{array}$ \\
\hline $\begin{array}{l}\text { Processo para } \\
\text { identificar }\end{array}$ & $\begin{array}{l}\text { Como ponderar os valores e preferências dos } \\
\text { decisores e a combinação singular dos recursos da } \\
\text { organização de forma a gerar vantagem competitiva } \\
\text { para a organização? }\end{array}$ \\
\hline Mensuração & \multirow{2}{*}{$\begin{array}{l}\text { Como utilizar escalas que atribuam níveis de } \\
\text { referência, ao mesmo tempo em que consideram as } \\
\text { propriedades da mensuração e ainda permitam a } \\
\text { integração de indicadores? }\end{array}$} \\
\hline Integração & \\
\hline Aperfeiçoamento & $\begin{array}{l}\text { Como analisar a organização estrategicamente, de } \\
\text { forma qualitativa e quantitativa, de modo a identificar } \\
\text { e organizar seus objetivos, definindo e priorizando as } \\
\text { ações com maior impacto? } \\
\text { Como valer-se do conhecimento gerado pelo } \\
\text { instrumento de avaliação e desempenho para construir } \\
\text { ações estratégicas que aperfeiçoarão os objetivos a } \\
\text { serem alcançados? }\end{array}$ \\
\hline
\end{tabular}

Fonte: Autores.

A primeira lente estudada, referente à abordagem, visa identificar as filiações teóricas utilizadas nos artigos. Nesse quesito observou-se que há espaço para mais trabalhos utilizando visões construtivistas de mundo, uma vez que somente o construtivismo tem a preocupação de expandir o entendimento do decisor de modo que ele entenda os efeitos do contexto nas questões que ele julga relevantes.

Com referência à singularidade, o objetivo é perceber como pode ser elaborado um modelo de avaliação de desempenho que consiga combinar tanto os pontos subjetivos dos decisores 
quanto os aspectos físicos e humanos do contexto em um modelo particular que visa ao apoio na gestão estratégica organizacional (Lacerda et. al., 2011a). Nesse sentido, a partir da visão construtivista abordada nesta pesquisa, entende-se que deveria haver mais estudos que reconhecessem a importância da singularidade dos contextos, pois somente eles trazem oportunidades de melhoria que consideram a combinação única dos recursos da empresa para gerar vantagens competitivas de difícil imitação pelos concorrentes.

A terceira análise realizada foi quanto à identificação e organização dos critérios utilizados. Nesse ponto, constatou-se que a maioria dos trabalhos usou literatura científica para essa definição e, considerando a consonância com o que foi exposto no escopo da singularidade, isso indica que o modelo proposto não está ponderando os valores e preferências do decisor e a combinação única dos recursos da organização, que é o que assegura as vantagens competitivas da organização.

A lente que avalia as formas de mensuração objetiva analisar a construção das escalas que representarão os objetivos estratégicos da organização, enquanto a lente da integração serve para mensurar o alcance dos objetivos em dado contexto, tanto de forma individual, quanto coletiva. A principal oportunidade identificada nesta análise diz respeito ao uso de escalas que permitam a integração dos indicadores, pois é essa visão macro que dará subsídio ao gerente para a tomada de decisão. Para atingir tal objetivo, precisarão ser consideradas as propriedades de mensuração (mencionadas no capítulo que analisou este critério) quando da definição da escala a ser usada; bem como a atribuição de níveis de referência em todas as escalas construídas.

A última lente analisada baseou-se na perspectiva do diagnóstico e do aperfeiçoamento da situação atual. Respectivamente, isso quer dizer: como realizar a análise estratégica de uma organização valendo-se de instrumentos qualitativos e quantitativos para identificar e organizar os objetivos e para criar e priorizar as ações com maior impacto global e sistêmico; e, como fazer uso do conhecimento gerado pelo instrumento de avaliação e desempenho para criar ações estratégicas que aperfeiçoarão o alcance dos objetivos estratégicos (Lacerda et. al., 2011a).

Neste quesito, identifica-se que existem oportunidades tanto no aspecto do diagnóstico quanto do aperfeiçoamento. No primeiro ponto, os trabalhos vigentes deixam a desejar quando analisam apenas os aspectos históricos - resultados passados - para inferir sobre possíveis efeitos futuros. Assim como já comentado em outras lentes, percebe-se que falta o reconhecimento do papel fundamental do decisor que precisa incorporar seus valores e preferência no contexto decisório. No segundo ponto, ainda são poucos os trabalhos que se 
preocupam em demonstrar como fazer uso do conhecimento gerado e isso, por si só, já se demonstra uma relevante oportunidade de estudo.

\section{Conclusões}

Primeiramente, cabe resgatar o objetivo primordial deste trabalho que foi dado como o propósito de identificar as oportunidades de pesquisa nos temas avaliação de desempenho e gerenciamento de projetos, a partir da análise de um conjunto de artigos científicos relevantes sobre esses assuntos.

Para atingir o objetivo proposto, foi necessária a realização de um trabalho que pode ser dividido em três etapas principais: a) execução de um processo estruturado para seleção de artigos com destaque no mundo acadêmico a partir de sua validação científica. Nesta fase foram identificados 17 trabalhos que atenderam os critérios definidos pelos pesquisadores; b) análise qualitativa e quantitativa deste portfólio. Essa atividade permitiu, entre outras informações, identificar os periódicos e autores relevantes nos assuntos pesquisados; e, c) realização de uma análise sistêmica nesses trabalhos, baseada em seis lentes que representam uma estrutura de valor proposta pelos autores. Essa última etapa construiu conhecimento nos pesquisadores de forma a identificarem oportunidades de estudo para futuras pesquisas que abordem conjuntamente os assuntos de avaliação de desempenho e gestão de projetos sob a ótica construtivista.

A partir da utilização do Proknow-C foi possível compor uma estrutura teórica científica validada para servir de embasamento a futuros estudos e pesquisas que estejam alinhados com os temas em questão, servindo assim como oportunidade para revisões de literatura e conhecimento do estado da arte nessas áreas de conhecimento (Oliveira et al., 2016). Vale mencionar que a aplicação de um método já cientificamente validado como o ProKnow-C para o atingimento desse objetivo é que dá segurança ao autor para saber que se está valendo de material cujas informações são realmente relevantes no contexto acadêmico.

A partir das lentes de análise utilizadas, para avaliação dos artigos mais relevantes ao tema, identifica-se a necessidade de pesquisas que reconheça a singularidade e valores e preferência do decisor, dentro da perspectiva construtivista. Assim, considera-se a importância de tais fatores frente aos critérios de avaliação para que o modelo desenvolvido possa ser legitimado, buscando não apenas informações externas ao contexto avaliado, ou seja, de critérios baseados na literatura, pois estes ignoram o conhecimento do decisor. 
Dessa forma, entende-se que este trabalho atinge seu objetivo de estimular novos estudos sobre os assuntos de avaliação de desempenho e gerenciamento de projetos, uma vez que demonstra lacunas na literatura vigente, identificadas através do processo de análise sistêmica.

Para encerrar, cabe salientar que as principais limitações deste trabalho dizem respeito ao uso das bases Web of Science (antiga ISI) e Scopus em abril de 2015 e também às percepções do pesquisador. Apesar de seguir uma metodologia que aqui foi detalhada, há etapas do processo em que a subjetividade do pesquisador é que determina os filtros utilizados e tal subjetividade é característica do caráter interpretativo da pesquisa (Paiva Júnior, Souza Leão \& Mello, 2011).

Uma das características deste trabalho se dá pelo seu alinhamento epistemológico construtivista, ou seja, onde a visão de mundo da pesquisa é não somente reconhecida, mas como se caracteriza um diferencial relevante. Assim, qualquer trabalho científico que desejar minimizar essa característica, estará sendo afiliado a uma vertente positivista, buscando critérios universais e o portfólio bibliográfico "ideal", que poderiam ser executados por sistemas computacionais e algoritmos determinísticos ou probabilísticos. Cabe a cada pesquisador definir o método que seja alinhado a sua visão de mundo, uma vez que a pesquisa científica é uma atividade puramente humana, social e cognitiva.

Como recomendações para pesquisas futuras, destaca-se que o Proknow-C, utilizado para análise bibliométrica, é um método dinâmico e está em constante busca de aperfeiçoamentos. Com isso, ressalta-se a importância da utilização de referências recentes e atualizadas do método em pesquisas futuras que visam utilizar este método.

\section{Referências}

Andersen, E. S.; Birchall, D.; Jessen, S. A.; \& Money, A. H. (2006). Exploring project success. Baltic Journal of Management, 1(2), 127-147.

Andersen, E. S. (2008). Rethinking project management: an organisational perspective. Strategic Direction, 26(3).

Ahmad, S. B.; Svalestuen, F.; Andersen, B.; \& Torp, O. (2016). A review of performance measurement for successful concurrent construction. Procedia-Social and Behavioral Sciences, 226, 447-454. 
Atkinson, R. (1999). Project management: cost, time and quality, two best guesses and a phenomenon, its time to accept other success criteria. International Journal of Project Management, 17(6), 337-342.

Barclay, C. (2008). Towards an integrated measurement of IS project performance: The project performance scorecard. Information Systems Frontiers, 10(3), 331-345.

Barclay, C. \& Osei-Bryson, K. M. (2010). Project performance development framework: An approach for developing performance criteria \& measures for information systems (IS) projects. International Journal of Production Economics, 124(1), 272-292.

Barney, J.; Wright, M.; Ketchen J.; \& David J. (2001). The resource-based view of the firm: Ten years after 1991. Journal of Management, 27, 625-641.

Barzilai, J. (2001). On the foundations of measurement. In: Systems, Man, and Cybernetics, IEEE International Conference on. IEEE, 2001. pp. 401-406.

Behn, R. D. (2003). Why measure performance? Different purposes require different measures. Public Administration Review, 63(5), 586-606.

Blindenbach-Driessen, F.; Van Dalen, J.; \& Van Den Ende, J. (2010). Subjective performance assessment of innovation projects. Journal of Product Innovation Management, 27(4), 572592.

Bryde, D. J. (2003). Modelling project management performance. International Journal of Quality \& Reliability Management, 20(2), 229-254.

Cao, Q. \& Hoffman, J. J. (2011). A case study approach for developing a project performance evaluation system. International Journal of Project Management, 29(2), 155-164.

Cervo, A. L.; Bervian, P. A.; Silva, R. da (2007). Metodologia científica (6a ed.). São Paulo: Pearson Prentice Hall. 
Charnes, A.; Cooper, W. W.; \& Rhodes, E. (1978). Measuring the efficiency of decision making units. European Journal of Operational Research, 2(6), 429-444.

Cooper, R.G. (2007). Managing technology development projects. IEEE Engineering International Journal of Operations \& Production Management, 21, p. 400.

Dienstmann, J. S.; Lacerda, R. T. D. O.; Ensslin, L.; \& Ensslin, S. R. (2014). Gestão da inovação e avaliação de desempenho: processo estruturado de revisão da literatura. Revista Produção Online, 14(1), 2-30.

Dweiri, F. \& Kablan, M. (2006). Using fuzzy decision making for the evaluation of the project management internal efficiency. Decision Support Systems, 42(2), 712-726.

Elsevier Scopus. 2015 (24), jun. 2015. p. Content.

Ensslin, L.; Ensslin, S. R.; Lacerda, R. T. D. O.; \& Tasca, J. E. (2010). ProKnow-C, knowledge development process-constructivist. Processo técnico com patente de registro pendente junto ao INPI. Brasil.

Ensslin, S. R.; Ensslin, L.; Lacerda, R. T. D. O.; \& Santos Matos, L. dos (2013). Evidenciação do estado da arte do tema avaliação do desempenho na regulação de serviços públicos segundo a percepção dos pesquisadores. Gestão Pública: Práticas e Desafios, 4(1).

Ensslin, L ; Back, F. T. E. E. ; Ensslin, S. R.; \& Lacerda, R. T. O. (2013). Improved decision aiding in human resource management: a case using constructivist multi-criteria decision aiding. The International Journal of Productivity and Performance Management, 62, 735757.

Ferreira, A. G. G. C. (2012). Bibliometria na avaliação de periódicos científicos-Bibliometrics in the evaluation of scientific journals. Pesquisa Brasileira em Ciência da Informação e Biblioteconomia, 5(2).

Gil, A. C. (1999). Pesquisa social: métodos e técnicas. São Paulo: Atlas, vol. 5. 
Google (2015). Google Scholar. 2015 (24), jun. 2015. p. About.

Hammond, K. R. \& Stewart, T. R. (2001). The essential brunswik: Beginnings, explications, applications. Oxford University Press.

Jha, K. N. \& Iyer, K. C. (2007). Commitment, coordination, competence and the iron triangle. International Journal of Project Management, 25(5), 527-540.

Jugdev, K.; Thomas, J.; \& Delisle, C.L. (2001). Rethinking project management: old truths and new insights. Project Management Forum, 7, 36-43.

Jugdev, K. \& Muller, R. (2006). A retrospective look at our evolving understanding of project success factors: a multivariate, typological approach. $R \& D$ Management, 32 .

Karlsen, J. T.; Andersen, J.; Birkely, L. S.; \& Odegard, E. (2005). What characterizes successful it projects. International Journal of Information Technology \& Decision Making, 4(4), 525-540.

Keeney, R. L. (1992). Value-focused thinking: A path to creative decision making. Harvard University Press.

Kennerley, M.; \& Neely, A. (2002). A framework of the factors affecting the evolution of performance measurement systems. International Journal of Operations \& Production Management, 22(11), 1222-1245.

Klijn, E. (2008). Governance and governance networks in Europe: An assessment of ten years of research on the theme. Public Management Review, 10, 505-525.

Knoff, L. C.; Lacerda, R. T. D. O.; Ensslin, L.; \& Ensslin, S. (2014). Mapeamento de publicações científicas sobre estratégia de manufatura: uma abordagem baseada em processos. Revista Produção Online, 14(2), 403-429. 
Lacerda, R. T. O.; Ensslin, L.; \& Ensslin, S. R. (2011a). Contribuições à gestão estratégica de organizações quando analisados na visão de seu desempenho. Gestão.Org-Revista Eletrônica de Gestão Organizacional, 9(2).

(2011b). A performance measurement view of IT project management. International Journal of Productivity and Performance Management, 60(2), 132-151.

- (2012). Uma análise bibliométrica da literatura sobre estratégia e avaliação de desempenho. Gestão \& Produção, 19(1).

(2014). Research opportunities in strategic management field: a performance measurement approach. International Journal of Business Performance Management, $15(2), 158-174$.

Lacerda, R. T. O; Ensslin, L.; Ensslin, S. R.; Knoff, L; \& Martins, C. J. D. (2016). Research opportunities in business process management and performance measurement from a constructivist view. Knowledge and Process Management, 23, 18-30.

Lacerda, R. T. O.; Klein, B.; Fulco, J.; Santos, G.; \& Bittarello, K. (2017). Integração inovadora entre empresas incubadas e universidades para geração contínua de vantagens competitivas em ambientes dinâmicos. Revista de Gestão e Tecnologia, 7(2), 78-96.

Lenfle, S. \& Loch, C. (2010). Lost roots: how project management came to emphasize control over flexibility and novelty. California. Management, 53, 32-55.

Ling, F. Y. Y.; Low, S. P.; Wang, S. Q.; \& Lim, H. H. (2009). Key project management practices affecting Singaporean firms' project performance in China. International Journal of Project Management, 27(1), 59-71.

Lipovetsky, S.; Tishler, A.; Dvir, D.; \& Shenhar, A. (1997). The relative importance of Project Success. Project Management Journal, 36, 19-31. 
Lowe, K. B. \& Gardner, W. L. (2001). Ten years of the leadership quarterly: Contributions and challenges for the future. The Leadership Quarterly,11, 459-514.

Marafon, A. D.; Ensslin, L.; Ensslin, S. R.; \& Lacerda, R. T. (2012). Revisão sistêmica da literatura sobre avaliação de desempenho na gestão de P\&D. Revista Gestão Industrial, $8(3)$.

. (2015). The effectiveness of multi-criteria decision aid methodology: A case study of R\&D management. European Journal of Innovation Management, 18(1), 86-109.

Marques, G.; Gourc, D.; \& Lauras, M. (2011). Multi-criteria performance analysis for decision making in project management. International Journal of Project Management, 29(8), 1057-1069.

Melão, N.; \& Pidd, M. (2000). A conceptual framework for understanding business processes and business process modelling. Information Systems Journal, 10(2), 105-129.

Mir, F. A. \& Pinnington, A. H. (2014). Exploring the value of project management: Linking Project Management Performance and Project Success. International Journal of Project Management, 32(2), 202-217.

Mota, C. M. D. M.; Almeida, A. T. de; \& Alencar, L. H. (2009). A multiple criteria decision model for assigning priorities to activities in project management. International Journal of Project Management, 27(2), 175-181.

Ngacho, C.; \& Das, D. (2015). A performance evaluation framework of construction projects: insights from literature. International Journal of Project Organisation and Management, 7(2), 151-173.

Nassar, N. \& Abourizk, S. (2014). Practical application for integrated performance measurement of construction projects. Journal of Management in Engineering, 30(6). 
Oliveira, L. V.; Lacerda, R, T. O.; Fiates, G. G. S. \& Ensslin, S. R. (2016). Avaliação de desempenho e gerenciamento de projetos: uma análise bibliométrica. Revista de Gestão e Projetos, [s.1.], 7(1), 95-113.

Packendorff, J. (1995). Inquiring into the temporary organization: new directions for project management research. Scand. J. Manag, 11, 319-333.

Paiva Júnior, F. G. de; Souza Leão, A. L. M. de; \& Mello, S. C. B. de. (2011). Validade e confiabilidade na pesquisa qualitativa em Administração. Ciências da Administração, 13(31), 190-209.

Pollack, J. (2007). The changing paradigms of project management. International Journal of Project Management, 25, 266-74.

Qureshi, T. M.; Warraich, A. S. \& Hijazi, S. T. (2009). Significance of project management performance assessment (PMPA) model. International Journal of Project Management, 27(4), 378-388.

Rosacker, K. M. \& Olson, D. L. (2008). An empirical assessment of it project selection and evaluation methods in state government. Project Management Journal, 39(1), 49-58.

Roy, B. (1993). Decision science or decision-aid science? European Journal of Operational Research, 66(2), 184-203.

Saaty, T. L. (2008). Decision making with the analytic hierarchy process. International Journal of Services Sciences, 1(1), 83-98.

Silva da Rosa, F.; Rolim Ensslin, S.; Ensslin, L. \& João Lunkes, R. (2012). Environmental disclosure management: a constructivist case. Management Decision, 50(6), 1117-1136.

Shenhar, A. \& Dvir, D. (2007). Reinventing project management: the diamond approach to successful growth and innovation. Harvard Business Press, Boston. 
Shenhar, A.J.; Tishler, A.; Dvir, D.; Lipovetsky, S. \& Lechler, T. (2002). Refining the search for success dimensions. $R \& D$ Management, 27, 97-106.

Svejvig, P. \& Andersen, P. (2015). Rethinking project management: A structured literature review with a critical look at the brave new world. International Journal of Project Management, 33(2), 278-290.

Taticchi, P.; Tonelli, F., \& Cagnazzo, L. (2010). Performance measurement and management: a literature review and a research agenda. Measuring Business Excellence, 14, 4-18.

Teece, D. J. (2007). Explicating dynamic capabilities: the nature and microfoundations of (sustainable) enterprise performance. Strategic Management Journal, 28(13), 1319-1350.

Thomas, G. \& Fernández, W. (2008). Success in IT projects: A matter of definition? International Journal of Project Management, 26(7), 733-742.

Tranfield, D. R.; Denyer, D. \& Smart, P. (2003). Towards a methodology for developing evidence-informed management knowledge by means of systematic review. British Journal of Management, 14, 207-222.

Tukel, O.I. \& Rom, W.O. (2001). An empirical investigation of project evaluation criteria.

Xu, Y. \& Yeh, C. H. (2014). A performance-based approach to project assignment and performance evaluation. International Journal of Project Management, 32(2), 218-228.

Wernerfelt, B. (1995). The resource- based view of the firm: Ten years after. Strategic Management Journal, 16, 171-174.

Willems, L. L.; \& Vanhoucke, M. (2015). Classification of articles and journals on project control and earned value management. International Journal of Project Management, 33(7), 1610-1634. 
Perspectivas de pesquisa sobre avaliação de desempenho e gerenciamento de projetos

Williams, T. (2005). Assessing and moving on from the dominant project management discourse in the light of project overruns. IEEE Transactions on Engineering Management, $52,497-508$

Winter, M.; Smith, C.; Morris, P.; \& Cicmil, S. (2006). Directions for future research in project management: The main findings of a UK government-funded research network. International Journal of Project Management, 24, 638-649. 